\title{
A Measure of Departure from Average Symmetry for Square Contingency Tables with Ordered Categories
}

\author{
Kouji Tahata, Kouji Yamamoto, Noriyuki Nagatani, and Sadao Tomizawa \\ Dept. of Information Sciences, Tokyo University of Science, Japan
}

\begin{abstract}
For the analysis of square contingency tables, Tomizawa (1994), Tomizawa, Seo, and Yamamoto (1998), and Tomizawa, Miyamoto, and Hatanaka (2001) considered measures to represent the degree of departure from symmetry. However, the maximum value of these measures cannot distinguish two kinds of complete asymmetry (say, complete-upper-asymmetry and complete-lower-asymmetry). The present paper proposes a measure which can distinguish two kinds of complete asymmetry for square tables with ordered categories. Especially the proposed measure is useful for representing the degree of departure from symmetry when the conditional symmetry model holds. Examples are given.

Zusammenfassung: Für die Analyse quadratischer Kontingenztafeln betrachteten Tomizawa (1994), Tomizawa et al. (1998), und Tomizawa et al. (2001) Maße, die den Grad der Abweichung von der Symmetrie bewerten. Der maximale Wert dieser Maße kann jedoch zwei Arten völliger Asymmetrie nicht unterscheiden (complete-upper-asymmetry und complete-lowerasymmetry). Im vorliegenden Aufsatz wird ein Maß vorgeschlagen, das zwei Arten völliger Asymmetrie für quadratische Tafeln mit geordneten Kategorien unterscheiden vermag. Das empfohlene Maß ist speziell nützlich um den Grad der Abweichung von der Symmetrie zu beschreiben falls das konditionale Symmetriemodell hält. Beispiele sind angeführt.
\end{abstract}

Keywords: Asymmetry, Conditional Symmetry, Ordinal Data.

\section{Introduction}

Consider an $R \times R$ square contingency table. Let $p_{i j}$ denote the probability that an observation will fall in the $i$ th row and $j$ th column of the table $(i=1, \ldots, R, j=1, \ldots, R)$. The symmetry model is defined by

$$
p_{i j}=p_{j i}, \quad(i \neq j),
$$

see, for example, Bowker (1948), Bishop, Fienberg, and Holland (1975, p. 282), and Agresti (2002, p. 424). This model states that the probability that an observation will fall in cell $(i, j), i \neq j$, is equal to the probability that it falls in symmetric cell $(j, i)$.

When the symmetry model does not hold, we may be interested in applying the extended symmetry models, e.g., the quasi-symmetry (Caussinus, 1965) and the marginal homogeneity (Stuart, 1955) models, and asymmetry models, e.g., the conditional symmetry (McCullagh, 1978) and the diagonals-parameter symmetry (Goodman, 1979) models. In addition, when the symmetry model does not hold, we are interested in measuring the 
degree of departure from the symmetry model. The measure is useful for comparing the degrees of departure from the model in several tables.

For square contingency tables with nominal categories, Tomizawa (1994) proposed the measures to represent the degree of departure from symmetry, which are expressed by using the Kullback-Leibler information and the Pearson $\chi^{2}$-type discrepancy. Tomizawa et al. (1998) considered a power-divergence-type measure $\Phi^{(\lambda)}$ which includes these measures. See Appendix for the measure $\Phi^{(\lambda)}$. Also, for square contingency tables with ordered categories, Tomizawa et al. (2001) proposed another power-divergence-type measure to represent the degree of departure from symmetry although the detail is omitted.

As described in the Appendix, assuming that $p_{i j}+p_{j i} \neq 0$, (i) the measure $\Phi^{(\lambda)}$ is between 0 and 1, (ii) $\Phi^{(\lambda)}=0$ if and only if the symmetry model holds, and (iii) $\Phi^{(\lambda)}=1$ if and only if the degree of departure from symmetry is maximum (say, complete asymmetry). That is, $p_{i j}=0$ (then $p_{j i}>0$ ) or $p_{j i}=0$ (then $p_{i j}>0$ ) for all $i<j$. However, using the measure $\Phi^{(\lambda)}$ (and using the Tomizawa et al., 2001 measure), we cannot distinguish two kinds of complete asymmetry, namely, that the complete asymmetry is which of (i) $p_{i j}=0$ (then $p_{j i}>0$ ) for all $i<j$, or (ii) $p_{j i}=0$ (then $p_{i j}>0$ ) for all $i<j$ (i.e., which of (i) all observations concentrate in the lower left triangle cells in the table, or (ii) those concentrate only in the upper right triangle cells). Since these two kinds of complete asymmetry indicate the opposite different maximum departures from symmetry, we are interested in proposing a measure which can take the different values for them.

The purpose of this paper is to propose such a measure which can distinguish two kinds of complete asymmetry for square contingency tables with ordered categories.

\section{A Measure}

Consider the $R \times R$ table with ordered categories. Assume that $p_{i j}+p_{j i} \neq 0$. Let

$$
\delta=\sum \sum_{s \neq t} p_{s t} \quad \text { and } \quad p_{i j}^{*}=\frac{p_{i j}}{\delta}
$$

for $i=1, \ldots, R, j=1, \ldots, R, i \neq j$. We shall consider a measure defined by

$$
\varphi=\frac{4}{\pi} \sum_{i<j} \sum_{i j}\left(p_{i j}^{*}+p_{j i}^{*}\right)\left(\theta_{i j}-\frac{\pi}{4}\right),
$$

where

$$
\theta_{i j}=\cos ^{-1}\left(\frac{p_{i j}}{\sqrt{p_{i j}^{2}+p_{j i}^{2}}}\right) .
$$

The range of $\theta_{i j}$ is $0 \leq \theta_{i j} \leq \pi / 2$. Thus, the measure $\varphi$ lies between -1 and 1 . The measure $\varphi$ has characteristics that (i) $\varphi=-1$ if and only if $p_{i j}>0$ for all $i<j$ (then $p_{j i}=0$ for all $i<j$ ), say, complete-upper-asymmetry, and (ii) $\varphi=1$ if and only if $p_{j i}>0$ for all $i<j$ (then $p_{i j}=0$ for all $i<j$ ), say, complete-lower-asymmetry. Also $\varphi=0$ indicates that the average of $\theta_{i j}-\pi / 4$ for $i<j$ equals zero on condition that an observation will fall in one of off-diagonal cells of the table. Therefore when $\varphi=0$, 
we shall refer to this structure as the average symmetry. Note that the average symmetry model cannot be defined by means of the probabilities $p_{i j}$. Also, we note that if the symmetry holds then the average symmetry holds, but the converse does not hold.

Using $\varphi$, we can see whether the average symmetry departs toward the completeupper-asymmetry or toward the complete-lower-asymmetry. As $\varphi$ approaches -1 , the departure from the average symmetry becomes greater toward complete-upper-asymmetry. As $\varphi$ approaches 1 , it becomes greater toward complete-lower-asymmetry.

\section{Relationship between $\varphi$ and Conditional Symmetry}

We next consider the relationship between the measure $\varphi$ and the conditional symmetry model. The conditional symmetry model (McCullagh, 1978) is defined by

$$
p_{i j}=\Delta p_{j i} \quad i<j .
$$

A special case of this model obtained by putting $\Delta=1$ is the symmetry model. If there is a structure of conditional symmetry in the table, then the measure $\varphi$ can be expressed as

$$
\varphi=\frac{4}{\pi} \cos ^{-1}\left(\frac{\Delta}{\sqrt{\Delta^{2}+1}}\right)-1 .
$$

Therefore, $\varphi=0$ if and only if the symmetry model holds, i.e., $\Delta=1$, thus $p_{i j}=p_{j i}$ for all $i \neq j$. As the value of $\Delta$ approaches the infinity, the measure $\varphi$ approaches -1 . As the value of $\Delta$ approaches zero, the $\varphi$ approaches 1 . Thus, for comparisons between several tables, if it can be estimated that there is a structure of conditional symmetry in each table, then the measure $\varphi$ would be adequate for representing and comparing the degree of departure from the symmetry toward the complete-upper-asymmetry and toward the complete-lower-asymmetry.

\section{Approximate Confidence Interval and Test}

Let $n_{i j}$ denote the observed frequency in the $i$ th row and $j$ th column of the table $(i=$ $1, \ldots, R, j=1, \ldots, R)$. Assuming that a multinomial distribution applies to the $R \times R$ table, we shall consider the approximate variance for the estimated measure and a largesample confidence interval for the measure $\varphi$ using the delta method, the descriptions of which are given by, e.g., Bishop et al. (1975, Sec. 14.6). The sample version of $\varphi$, i.e., $\hat{\varphi}$, is given by $\varphi$ with $p_{i j}$ replaced by $\hat{p}_{i j}$, where $\hat{p}_{i j}=n_{i j} / n$ and $n=\sum \sum n_{i j}$.

Let $\hat{p}$ be the $R^{2} \times 1$ vector

$$
\hat{p}=\left(\hat{p}_{11}, \hat{p}_{12}, \ldots, \hat{p}_{1 R}, \hat{p}_{21}, \ldots, \hat{p}_{R R}\right)^{\prime},
$$

where "prime" denotes the transpose. Also, let us define the vector $p$ in terms of the $p_{i j}$ 's in the same way as $\hat{p}$. Then $\sqrt{n}(\hat{p}-p)$ is asymptotically distributed as normal with mean zero and variance $\Sigma(p)$, where $\Sigma(p)=\operatorname{diag}(p)-p p^{\prime}$ and $\operatorname{diag}(p)$ denotes a diagonal matrix with the $i$ th element of $p$ as the $i$ th diagonal element. We obtain

$$
\hat{\varphi}=\varphi+d(p)(\hat{p}-p)+o(\|\hat{p}-p\|),
$$


where $d(p)=\partial \varphi / \partial p^{\prime}$ being a $1 \times R^{2}$ vector. Then $\sqrt{n}(\hat{\varphi}-\varphi)$ follows asymptotically (as $n \rightarrow \infty)$ a normal distribution with mean zero and variance,

$$
\operatorname{var}(\varphi)=d(p) \Sigma(p) d(p)^{\prime}=\sum_{i<j} \sum_{i j}\left(p_{i j} D_{i j}^{2}+p_{j i} D_{j i}^{2}\right)
$$

where for $i<j$

$$
\begin{aligned}
& D_{i j}=\frac{4}{\pi \delta}\left\{\cos ^{-1}\left(\frac{p_{i j}}{\sqrt{p_{i j}^{2}+p_{j i}^{2}}}\right)-\frac{p_{j i}\left(p_{i j}+p_{j i}\right)}{p_{i j}^{2}+p_{j i}^{2}}\right\}-\frac{\varphi+1}{\delta}, \\
& D_{j i}=\frac{4}{\pi \delta}\left\{\cos ^{-1}\left(\frac{p_{i j}}{\sqrt{p_{i j}^{2}+p_{j i}^{2}}}\right)+\frac{p_{i j}\left(p_{i j}+p_{j i}\right)}{p_{i j}^{2}+p_{j i}^{2}}\right\}-\frac{\varphi+1}{\delta} .
\end{aligned}
$$

Let $\widehat{\operatorname{var}}(\varphi)$ denote $\operatorname{var}(\varphi)$ with $p_{i j}$ replaced by $\hat{p}_{i j}$. Thus, the square root of $\widehat{\operatorname{var}}(\varphi) / n$ is an estimated standard error of $\hat{\varphi}$, and

$$
\hat{\varphi} \pm z_{\alpha / 2} \sqrt{\widehat{\operatorname{var}}(\varphi) / n}
$$

is an approximate $100(1-\alpha) \%$ confidence interval for $\varphi$, where $z_{\alpha / 2}$ is the percentage point of the standard normal distribution corresponding to a two-tail probability of $\alpha$.

The maximum likelihood estimates of expected frequencies under each of the symmetry and conditional symmetry models are given as the closed-form, and those under the average symmetry model can be obtained using the Newton-Raphson method to the loglikelihood equation although the details are omitted. The symmetry, conditional symmetry and average symmetry models can be tested for goodness-of-fit by, e.g., the likelihood ratio chi-squared statistic with $R(R-1) / 2,(R+1)(R-2) / 2$ and 1 degrees of freedom, respectively.

\section{Examples}

Example 1: Consider the data in Table 1a taken from Stuart (1955). These are data on unaided distance vision of 7477 women aged 30 to 39 employed in Royal Ordnance factories in Britain from 1943 to 1946.

We see from Table 2 that for the data in Table 1a, the estimated value of the measure $\varphi$ is -0.0934 and all values in the confidence interval for $\varphi$ are negative. Therefore, the average symmetry for the women's right and left eyes departs toward the completeupper-asymmetry. Table 3 gives the values of likelihood ratio chi-squared statistic for testing goodness-of-fit of each model. We see from Table 3 that each model of symmetry and average symmetry fits the data in Table 1a poorly, but the conditional symmetry model fits these data well. So we can see from the estimated measure that the degree of departure from symmetry for the vision data in Table 1a is estimated to be 9.3 percent of the maximum departure toward the complete-upper-asymmetry which indicates that the right eye is better than her left eye for all women when we define the maximum departure toward it as 100 percent. 
Table 1: Unaided vision data of (a) 7477 women in Britain (Stuart, 1955), (b) 3242 men in Britain (Stuart, 1953), and (c) 4746 students in Japan (Tomizawa, 1984).

(a) Women in Britain

\begin{tabular}{lrrrrr}
\hline Right eye & \multicolumn{4}{c}{ Left eye grade } & \\
\cline { 2 - 5 } \multicolumn{1}{c}{ grade } & Best (1) & Second (2) & Third (3) & Worst (4) & Total \\
\hline Best (1) & 1520 & 266 & 124 & 66 & 1976 \\
Second (2) & 234 & 1512 & 432 & 78 & 2256 \\
Third (3) & 117 & 362 & 1772 & 205 & 2456 \\
Worst (4) & 36 & 82 & 179 & 492 & 789 \\
\hline Total & 1907 & 2222 & 2507 & 841 & 7477 \\
\hline
\end{tabular}

(b) Men in Britain

\begin{tabular}{lrrrrr}
\hline Right eye & \multicolumn{4}{c}{ Left eye grade } & \\
\cline { 2 - 5 } \multicolumn{1}{c}{ grade } & Best (1) & Second (2) & Third (3) & Worst (4) & Total \\
\hline Best (1) & 821 & 112 & 85 & 35 & 1053 \\
Second (2) & 116 & 494 & 145 & 27 & 782 \\
Third (3) & 72 & 151 & 583 & 87 & 893 \\
Worst (4) & 43 & 34 & 106 & 331 & 514 \\
\hline Total & 1052 & 791 & 919 & 480 & 3242 \\
\hline
\end{tabular}

(c) Students in Japan

\begin{tabular}{lrrrrr}
\hline Right eye & \multicolumn{4}{c}{ Left eye grade } & \\
\cline { 2 - 5 } \multicolumn{1}{c}{ grade } & Best (1) & Second (2) & Third (3) & Worst (4) & Total \\
\hline Best (1) & 1291 & 130 & 40 & 22 & 1483 \\
Second (2) & 149 & 221 & 114 & 23 & 507 \\
Third (3) & 64 & 124 & 660 & 185 & 1033 \\
Worst (4) & 20 & 25 & 249 & 1429 & 1723 \\
\hline Total & 1524 & 500 & 1063 & 1659 & 4746 \\
\hline
\end{tabular}

Table 2: The estimates of $\varphi$, estimated approximate standard errors of $\hat{\varphi}$, and approximate $95 \%$ confidence intervals for $\varphi$, applied to the data in Table 1a, $1 \mathrm{~b}$ and $1 \mathrm{c}$.

\begin{tabular}{crcc}
\hline $\begin{array}{c}\text { Applied } \\
\text { data }\end{array}$ & $\begin{array}{c}\text { Estimated } \\
\text { measure }\end{array}$ & $\begin{array}{c}\text { Standard } \\
\text { error }\end{array}$ & $\begin{array}{c}\text { Confidence } \\
\text { interval }\end{array}$ \\
\hline Table 1a & -0.0934 & 0.0270 & $(-0.1462,-0.0405)$ \\
Table 1b & 0.0388 & 0.0398 & $(-0.0392,+0.1169)$ \\
Table 1c & 0.1291 & 0.0369 & $(+0.0568,+0.2014)$ \\
\hline
\end{tabular}

Table 3: Likelihood ratio statistics for the symmetry, average symmetry and conditional symmetry model. The parenthesized values are the respective degrees of freedom.

\begin{tabular}{cccr}
\hline $\begin{array}{c}\text { Applied } \\
\text { data }\end{array}$ & Symmetry & $\begin{array}{c}\text { Average } \\
\text { symmetry }\end{array}$ & $\begin{array}{c}\text { Conditional } \\
\text { symmetry }\end{array}$ \\
\hline Table 1a & $19.25^{*}(6)$ & $11.85^{*}(1)$ & $7.35(5)$ \\
Table 1b & $4.77(6)$ & $0.95(1)$ & $3.82(5)$ \\
Table 1c & $16.95^{*}(6)$ & $11.97^{*}(1)$ & $4.98(5)$ \\
\hline
\end{tabular}

$*$ means significant at the 0.05 level. 


\section{Example 2:}

Consider the data in Table $1 \mathrm{~b}$ taken from Stuart (1953). These are data on unaided distance vision of 3242 men in Britain.

We see from Table 2 that for the data in Table $1 \mathrm{~b}$, the estimated value of measure $\varphi$ is 0.0388 and the confidence interval for $\varphi$ includes zero. So this would indicate that there is a structure of average symmetry in the data in Table 1b. We also see from Table 3 that the symmetry model fits these data well, and each model of average symmetry and conditional symmetry also fits these data well. Therefore, it is estimated that there is a structure of symmetry for the data in Table $1 \mathrm{~b}$, and also the estimated measure $\hat{\varphi}$ would indicate it.

\section{Example 3:}

Consider the data in Table 1c taken from Tomizawa (1984). These are data on unaided distance vision of 4746 students aged 18 to about 25 including about $10 \%$ women in Faculty of Science and Technology, Science University of Tokyo in Japan examined in April 1982.

We see from Table 2 that for the data in Table 1c, the estimated value of measure $\varphi$ is 0.1291 and all values in the confidence interval for $\varphi$ are positive. Therefore, the average symmetry for the students' right and left eyes departs toward the complete-lowerasymmetry. This is a contrast to the women's vision data in Table 1a. We see from Table 3 that each model of symmetry and average symmetry fits the data in Table 1c poorly, but the conditional symmetry model fits these data well. So we can see from the estimated measure that the degree of departure from symmetry for the vision data in Table 1c is estimated to be 12.9 percent of the maximum departure toward the completelower-asymmetry which indicates that the left eye is better than his/her right eye for all students.

In addition, when we compare the data in Tables 1a and 1c using the estimated measure $\hat{\varphi}$, the degree of departure from the symmetry for the right and left eyes is greater in the students data in Table 1c than in the women data in Table 1a (see Table 2). Since $\hat{\varphi}$ is negative for the women vision data and positive for the students vision data, a woman's right eye tends to be greater than her left eye, and a student's left eye tends to be greater than his/her right eye.

\section{Concluding Remarks}

The proposed measure $\varphi$ is useful for representing what degree the departure from the average symmetry is toward two kinds of complete asymmetry (i.e., the complete-upperasymmetry and the complete-lower-asymmetry). The measure $\varphi$ can distinguish these two kinds of complete asymmetry although the measures in Tomizawa (1994), Tomizawa et al. (1998), and Tomizawa et al. (2001) cannot distinguish them.

Since $\hat{\varphi}$ lies between -1 and 1 without the dimension and the sample size, $\hat{\varphi}$ would be useful for comparing the degrees of departure from the average symmetry in several tables.

Especially, if it can be estimated that there is a structure of conditional symmetry in the table, then $\hat{\varphi}$ would be adequate for representing the degrees of departure from symmetry 
toward two kinds of complete asymmetry.

The measure $\varphi$ should be applied to the ordinal data of square tables with the same row and column classifications because $\varphi$ is not invariant under arbitrary similar permutations of row and column categories.

\section{Acknowledgments}

The authors would like to express their thanks to a referee for the helpful comments.

\section{Appendix}

For an $R \times R$ table with nominal categories, the measure of departure from symmetry considered by Tomizawa et al. (1998) are given as follows: assuming that $p_{i j}+p_{j i} \neq 0$, for $\lambda>-1$,

$$
\Phi^{(\lambda)}=\frac{\lambda(\lambda+1)}{2^{\lambda}-1} \sum_{i<j} \sum_{i j}\left(p_{i j}^{*}+p_{j i}^{*}\right) I_{i j}^{(\lambda)},
$$

where

$$
p_{i j}^{*}=\frac{p_{i j}}{\delta}, \quad \delta=\sum \sum_{s \neq t} p_{s t}, \quad p_{i j}^{c}=\frac{p_{i j}}{p_{i j}+p_{j i}}
$$

and

$$
I_{i j}^{(\lambda)}=\frac{1}{\lambda(\lambda+1)}\left(\left(p_{i j}^{c}\left(2 p_{i j}^{c}\right)^{\lambda}-1\right)+\left(p_{j i}^{c}\left(2 p_{j i}^{c}\right)^{\lambda}-1\right)\right)
$$

and the value at $\lambda=0$ is taken to be the limit as $\lambda \rightarrow 0$. Especially, $\Phi^{(0)}$ and $\Phi^{(1)}$ are two kinds of measures of the departure from symmetry, considered earlier by Tomizawa (1994).

Note that (i) $0 \leq \Phi^{(\lambda)} \leq 1$, (ii) $\Phi^{(\lambda)}=0$ if and only if the symmetry model holds, i.e., $p_{i j}=p_{j i}$, and (iii) $\Phi^{(\lambda)}=1$ if and only if $p_{i j}=0\left(\right.$ then $\left.p_{j i}>0\right)$ or $p_{j i}=0\left(\right.$ then $\left.p_{i j}>0\right)$ for $i<j$.

\section{References}

Agresti, A. (2002). Categorical Data Analysis (2nd ed.). New York: John Wiley \& Sons. Bishop, Y. M. M., Fienberg, S. E., and Holland, P. W. (1975). Discrete Multivariate Analysis: Theory and Practice. Cambridge, Massachusetts: The MIT Press.

Bowker, A. H. (1948). A test for symmetry in contingency tables. Journal of the American Statistical Association, 43, 572-574.

Caussinus, H. (1965). Contribution à l'analyse statistique des tableaux de corrélation. Annales de la Faculté des Sciences de l'Université de Toulouse, 29, 77-182.

Goodman, L. A. (1979). Multiplicative models for square contingency tables with ordered categories. Biometrika, 66, 413-418.

McCullagh, P. (1978). A class of parametric models for the analysis of square contingency tables with ordered categories. Biometrika, 65, 413-418. 
Stuart, A. (1953). The estimation and comparison of strengths of association in contingency tables. Biometrika, 40, 105-110.

Stuart, A. (1955). A test for homogeneity of the marginal distributions in a two-way classification. Biometrika, 42, 412-416.

Tomizawa, S. (1984). Three kinds of decompositions for the conditional symmetry model in a square contingency table. Journal of the Japan Statistical Society, 14, 35-42.

Tomizawa, S. (1994). Two kinds of measures of departure from symmetry in square contingency tables having nominal categories. Statistica Sinica, 4, 325-334.

Tomizawa, S., Miyamoto, N., and Hatanaka, Y. (2001). Measure of asymmetry for square contingency tables having ordered categories. Australian and New Zealand Journal of Statistics, 43, 335-349.

Tomizawa, S., Seo, T., and Yamamoto, H. (1998). Power-divergence-type measure of departure from symmetry for square contingency tables that have nominal categories. Journal of Applied Statistics, 25, 387-398.

Authors' address:

Kouji Tahata, Kouji Yamamoto, Noriyuki Nagatani, and Sadao Tomizawa

Department of Information Sciences

Faculty of Science and Technology

Tokyo University of Science

Noda City, Chiba 278-8510

Japan

E-Mail: kouji_tahata@is.noda.tus.ac.jp, yamamoto_kouji@ymail.plala.or.jp, tomizawa@is.noda.tus.ac.jp 